\title{
Protective effects of thoracic epidural anesthesia on hypoxia-induced acute lung injury in rabbits
}

\author{
LIJUN WANG ${ }^{1}$, JING $\mathrm{CANG}^{2}$ and $\mathrm{ZHANGGANG} \mathrm{XUE}{ }^{2}$ \\ ${ }^{1}$ Department of Anesthesiology, Ear, Eye, Nose and Throat Hospital, Fudan University, Shanghai 200031; \\ ${ }^{2}$ Department of Anesthesiology, Zhongshan Hospital, Fudan University, Shanghai 200032, P.R. China
}

Received October 16, 2014; Accepted October 7, 2015

DOI: 10.3892/etm.2016.3151

\begin{abstract}
The mechanism underlying the effect of thoracic epidural anesthesia (TEA) on hypoxia-induced acute lung injury (ALI) is currently unknown. In the present study, a rabbit acute lung injury model was established to investigate the effects of TEA on inflammatory factors, pulmonary surfactant and ultrastructure. A total of 56 rabbits were randomly assigned to four groups ( $\mathrm{n}=14$ per group): Control group (Group C), hypoxia group (Group H), sevoflurane group (Group S) and combined sevoflurane-epidural anesthesia group (Group ES). The ALI model was considered to have been successfully induced when the ratio of arterial oxygen partial pressure to fractional inspired oxygen was $<300$. The correct placement of a catheter for TEA was confirmed using epidurography. ALI was maintained for $3 \mathrm{~h}$. Arterial blood samples were collected from all groups during spontaneous breathing $\left(\mathrm{T}_{0}\right)$ and at $3 \mathrm{~h}$ after ALI induction $\left(\mathrm{T}_{5}\right)$ in order to evaluate the serum levels of interleukin (IL)-6, IL-8 and IL-10. Bronchoalveolar lavage fluid was harvested to determine the total phospholipid, saturated phosphatidylcholine and total protein levels. Furthermore, the dry/wet weight ratio and the mRNA expression levels of IL-6, IL-8 and IL-10 in the lung tissue were determined using ELISA. In addition, light and transmission electron microscopy and histological techniques were used to examine the morphology of alveolar type II cells in the rat lung tissue. The results indicate that changes of serum IL-6, IL-8 and IL-10 levels following ALI were consistent with the changes in the mRNA expression levels of IL-6, IL-8 and IL-10 in the lung tissue. TEA attenuated these changes and thus reduced the severity of the ALI. In addition, TEA improved the alveolar structure, reduced the number of polymorphonuclear cells and mitigated the damage of lamellar bodies. In summary, the results of the present study indicate
\end{abstract}

Correspondence to: Professor Jing Cang, Department of Anesthesiology, Zhongshan Hospital, Fudan University, 180 Fenglin Road, Shanghai 200032, P.R. China

E-mail: jingcangdoc@163.com

Key words: anesthesia, epidural, respiratory distress syndrome, inflammation, pulmonary surfactants, rabbit that TEA reduces lung tissue damage by inhibiting systemic and local inflammation, decreasing the inactivation of pulmonary surfactant and improving the alveolar ultrastructure following ALI.

\section{Introduction}

Acute lung injury (ALI) is a life-threatening disease that is characterized by acute onset, pulmonary inflammation, edema due to increased vascular permeability and severe hypoxemia (1). The incidence of ALI in the USA is 79/100,000, with an overall mortality count that is comparable to that of breast cancer (2). A previous study has demonstrated that lung injury caused by direct factors, including hypoxaemia and trauma, results in significant alveolar collapse, damage and edema (3). Acute hypoxia is a crucial cause of ALI, which may cause the body to produce extensive vasoconstriction, including a series of complicated changes to the internal environment (4).

In ALI, the inflammatory response to a primary infection in the lungs or systemic inflammation is mediated by a complex network of cytokines (5). Levels of proinflammatory cytokines, such as interleukin (IL)-1 $\beta$, tumor necrosis factor (TNF)- $\alpha$, IL-6 and IL-8, and anti-inflammatory cytokines, such as IL-1 receptor antagonist, IL-10 and IL-13, are elevated in the plasma or bronchoalveolar lavage fluid (BALF) in cases of ALI, indicating that a balance of these mediators determines the development of ALI (6-8).

Pulmonary surfactant consists of phospholipids and surfactant-specific proteins that determine the ability of a surfactant to reduce surface tension and thereby prevent alveolar collapse. Phospholipids are released from the lamellar bodies of alveolar type II cells and interact with surfactant proteins to form large aggregates. When alveolar type II cells are damaged, the synthesis and recirculation of surfactant is reduced (9).

Sevoflurane is among the most commonly used general anesthetics in clinical practice. Schwarzkopf et al (10) hypothesized that inhaled anesthetics may inhibit hypoxic pulmonary vasoconstriction and aggravate pulmonary shunt, interfere with alveolar type II epithelial cell metabolism and reduce the production of pulmonary surfactants. Therefore, sevoflurane may aggravate lung injury. In recent years, thoracic epidural anesthesia (TEA) has been widely used in major cardiac surgery (11). Compared with general anesthesia alone, general 
anesthesia combined with TEA may reduce respiratory system complications (12). However, the influence of TEA on hypoxia-induced ALI remains unclear.

In the present study, it was hypothesized that TEA would exert a protective effect against hypoxia-induced lung injury. In order to investigate this hypothesis, an ALI model was established in rabbits and the impact of TEA on a number of inflammatory factors was evaluated, including the serum concentrations of IL-6, IL-8 and IL-10. Furthermore, the mRNA expression levels of IL-6, IL-8 and IL-10 in the rabbit lung tissues were quantified. Finally, the impact of ALI and TEA on pulmonary surfactants and the ultrastructure in the rat lung tissue were investigated using microscopy and histological techniques.

\section{Materials and methods}

Animals and sampling. This study was conducted in strict accordance with the recommendations in the Guide for the Care and Use of Laboratory Animals of the National Institutes of Health (Eighth Edition, 2011). The animal use protocol was reviewed and approved by the Institutional Animal Care and Use Committee of Fudan University (Shanghai, China). A total of 56 New Zealand male white rabbits (age, 4-5 months old; weight, $2-3 \mathrm{~kg}$ ) were provided by the Animal Center of Fudan University. Anesthesia was induced with a $30-\mathrm{mg} / \mathrm{kg}$ intravenous dose of pentobarbital (P3761; Sigma-Aldrich, St. Louis, MO, USA). Following tracheotomy and intubation, the right carotid artery was cannulated for blood sampling. The auricular vein was cannulated for the administration of fluids and drugs.

Epidural catheter insertion for TEA. The first thoracic spinous process of the rabbits was upright and rapidly located. An epidural catheter was inserted by removing the fifth or sixth thoracic spinous process by vertical needling and was advanced $1 \mathrm{~cm}$ cephalad into the epidural space. Subsequently, $0.3 \mathrm{ml}$ contrast medium (60\% Conray; Shanghai Sangon Biological Engineering Co., Ltd., Shanghai, China) was injected into the epidural space and 16-slice computed tomography (GE Healthcare Bio-Sciences, Pittsburgh, PA, USA) and epidurography was performed to confirm catheterization.

Grouping and study protocol. The rabbits were allocated at random into four groups ( $\mathrm{n}=14$ per group): Control group (Group C), hypoxia group (Group H), sevoflurane group (Group S) and the combined sevoflurane-epidural anesthesia group (Group ES). Group ES received $3 \mathrm{mg} / \mathrm{kg} 2 \%$ lidocaine (070310; Shanghai Fuda Pharmaceutical Co., Ltd, Shanghai, China) and Groups $\mathrm{C}, \mathrm{H}$ and $\mathrm{S}$ received an identical volume of saline injected via the epidural catheter, followed by the injection of an identical dose every $1 \mathrm{~h}$. All animals maintained spontaneous breathing [fraction of inspired oxygen $\left.\left(\mathrm{FiO}_{2}\right), 21 \%\right]$ and were then mechanically ventilated using an ALC-V8 Rodent Ventilator (Shanghai Alcott Biotech Co., Shanghai, China). For Groups H, S and ES, the proportion of $\mathrm{O}_{2}$ in $\mathrm{N}_{2}$ was $14 \%$; and for Group $\mathrm{C}$ the $\mathrm{FiO}_{2}$ was $21 \%$. For all four groups, the tidal volume was $10-12 \mathrm{ml} / \mathrm{kg}$, the respiratory rate was $25 \mathrm{bpm}$ and the inspiratory-to-expiratory time ratio was 1:2. Sevoflurane (00784 S046F712; Baxter International Inc., Deerfield, IL, USA) was administered to the rabbits in Groups S and ES via inhalation at 1 minimum alveolar concentration using a Capnomac Ultima Anesthesia Monitor (Datex Ohmeda Oy, Helsinki, Finland). Anesthesia was maintained with $0.6 \mathrm{mg} / \mathrm{kg} / \mathrm{h}$ vecuronium (250393; Organon Pharmaceutical Co. Ltd., Nanjing, China) and $10 \mathrm{ml} / \mathrm{kg} / \mathrm{h}$ saline.

Establishment of the ALI model. The ALI model was considered to have been successfully induced after an arterial oxygen partial pressure $\left(\mathrm{PaO}_{2}\right) / \mathrm{FiO}_{2}$ value of $<300$ was obtained. Blood gas analysis was performed every 5 min using the i-STAT blood gas analysis system (Baxter Healthcare Corporation, Bloomington, IN, USA) following hypoxia until ALI was induced. At $3 \mathrm{~h}$ after reaching the ALI standard, the experiment was terminated. Blood gas analysis was performed during spontaneous breathing $\left(\mathrm{T}_{0}\right.$, baseline) and at 15,30,60, 120 and 180 min following ALI induction ( $T_{1-5}$, respectively) in Groups $\mathrm{H}, \mathrm{S}$ and ES, and at the corresponding time of mechanical ventilation in Group C.

Detection of inflammatory factors and mRNA expression. At $\mathrm{T}_{0}$ and $\mathrm{T}_{5}, 5 \mathrm{ml}$ blood samples were obtained and centrifuged for $10 \mathrm{~min}$ at $700 \mathrm{x} \mathrm{g}$. The serum was stored at $-70^{\circ} \mathrm{C}$ for subsequent evaluation of the levels of IL-6, IL-8 and IL-10. After completing these steps, blood was extracted from the right carotid arterial catheter, whilst anaesthetized, until the animals died. The chest wall was then opened and the lungs were excised. Pieces of lung $(1 \times 1 \mathrm{~cm})$ were extracted and homogenized in order to measure the mRNA expression levels of IL-6, IL-8 and IL-10 using reverse transcription-quantitative polymerase chain reaction analysis, as previously described (13) using an ABI 7300 Real-Time PCR system (Thermo Fisher Scientific, Inc., Waltham, MA, USA). Primers were synthesized by Shanghai Sangon Biological Engineering Co., Ltd., as follows: IL-6, upstream 5'-GAG AAAAGAGTTGTGCAATGGC-3' and downstream 5'-ACT AGGTTTGCCGAGTAGACC-3'; IL-8, upstream 5'-TTTGGA GCAGAGAGGAGGCAATG-3' and downstream 5'-ACCACA ATTCTGTCTTTCACGGGG-3'; and IL-10, upstream 5'-CAT GCCTGGCTCAGCACTGC-3' and downstream 5'-GGGAAC TGAGGTATCAGAGG-3'. Thermal cycling was performed as follows, $95^{\circ} \mathrm{C}$ for $15 \mathrm{~min}$ followed by 40 cycles of $94^{\circ} \mathrm{C}$ for $15 \mathrm{~s}$ and $60^{\circ} \mathrm{C}$ for $60 \mathrm{~s}$. Rabbit enzyme-linked immunosorbent assay (ELISA) kits were used to determine the serum concentrations of IL-6 (YS25008) IL-8 (YS25011) and IL-10 (YS25012; R\&D Systems, Inc., Minneapolis, MN, USA).

Measurement of lung dry/wet weight $(D / W)$ ratio and histological examination. Wet weights of some lung samples $(0.5 \times 0.5 \mathrm{~cm})$ were measured and the samples were then placed in a thermostatic incubator at $60^{\circ} \mathrm{C}$ for $48 \mathrm{~h}$ for measurement of the dry weight (PYX-DHS-500; Shanghai Yuejin Medical Instruments Co., Ltd., Shanghai, China). Other samples were fixed in glutaraldehyde (Sigma-Aldrich) and cut into $6 \mu \mathrm{m}$ sections prior to examination using a DVM6 optical microscope (Leica Microsystems GmbH, Wetzlar, Germany) and H-600 transmission electron microscope (Hitachi, Ltd., Tokyo, Japan).

Evaluation of BALF. Bronchoalveolar lavage was performed twice consecutively with $50 \mathrm{ml} 0.9 \% \mathrm{NaCl}$ each time. The 
Table I. Changes in $\mathrm{PaO}_{2}$ in the four groups (mmHg).

\begin{tabular}{|c|c|c|c|c|c|c|}
\hline Group & $\mathrm{T}_{0}$ & $\mathrm{~T}_{1}$ & $\mathrm{~T}_{2}$ & $\mathrm{~T}_{3}$ & $\mathrm{~T}_{4}$ & $\mathrm{~T}_{5}$ \\
\hline $\mathrm{C}$ & $115.4 \pm 39.7$ & $84.5 \pm 18.8$ & $101.2 \pm 20.7$ & $106.3 \pm 19.9$ & $121.5 \pm 9.2$ & $122.7 \pm 30.1$ \\
\hline $\mathrm{H}$ & $115.7 \pm 29.2$ & $29.5 \pm 4.4$ & $27.6 \pm 8.6$ & $30.5 \pm 8.9$ & $30.4 \pm 9.4$ & $32.8 \pm 4.1$ \\
\hline S & $94.1 \pm 11.6$ & $25 \pm 3.3$ & $29.4 \pm 1.5$ & $34.2 \pm 4.1$ & $34.1 \pm 2.9$ & $32.3 \pm 3.5$ \\
\hline $\mathrm{ES}$ & $86.1 \pm 6.1$ & $33.3 \pm 6.7$ & $34.5 \pm 6.32$ & $33.2 \pm 4.9$ & $38.7 \pm 5.2$ & $39.2 \pm 3.5$ \\
\hline
\end{tabular}

Data are presented as the mean \pm standard deviation. $C$, control group; $H$, hypoxia group; $S$, sevoflurane group; ES, combined sevoflurane-epidural anesthesia group; $\mathrm{T}_{0}$, spontaneous breathing; $\mathrm{T}_{1-5}, 15,30,60,120$ and $180 \mathrm{~min}$, respectively, after acute lung injury was induced in Groups $\mathrm{H}$, $\mathrm{S}$ and $\mathrm{ES}$, and at the corresponding time of mechanical ventilation in Group C. $\mathrm{PaO}_{2}$, arterial oxygen partial pressure.

$\mathbf{A}$

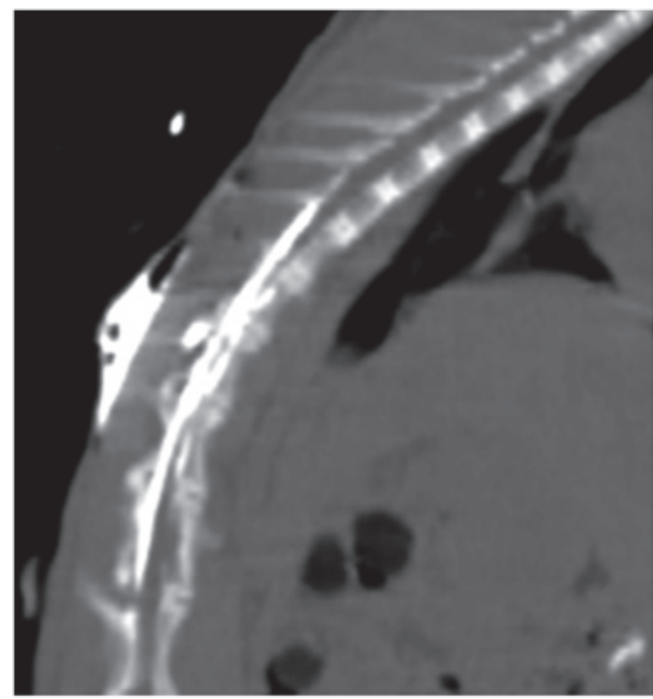

B

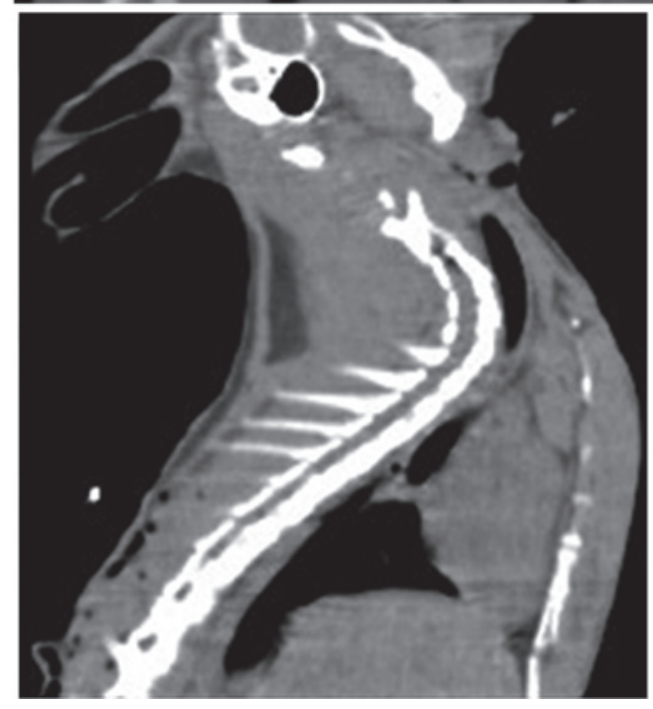

Figure 1. Epidurography results. Epidural imaging following the injection of contrast agent, confirming the presence of the epidural catheter in the epidural space. (A) Amplifying power, 120\%; resolution, 512x168. (B) Amplifying power, $100 \%$; resolution, $512 \times 168$.

fluid was instilled via the trachea into the lungs and immediately withdrawn. Following centrifugation in a cytocentrifuge for $10 \mathrm{~min}$ at $500 \mathrm{x} \mathrm{g}(80-2 \mathrm{~B}$; Shanghai Anting Scientific Instrument Factory, Shanghai, China), the supernatant fluid (BALF) was frozen and stored at $-70^{\circ} \mathrm{C}$ in a deep cryogenic refrigerator (Sanyo Electric Co., Ltd., Tokyo Japan) for further
Table II. Serum cytokine concentration changes following ALI induction.

\begin{tabular}{lclll}
\hline Group & Time & \multicolumn{1}{c}{ IL-6 } & IL-8 & IL-10 \\
\hline $\mathrm{C}$ & $\mathrm{T}_{0}$ & $49.4 \pm 17.6$ & $43.1 \pm 14.2$ & $39.5 \pm 18.7$ \\
& $\mathrm{~T}_{5}$ & $49.1 \pm 11.1$ & $52.5 \pm 20.8$ & $30.2 \pm 10.6$ \\
$\mathrm{H}$ & $\mathrm{T}_{0}$ & $33.8 \pm 5.7$ & $29.4 \pm 6.1$ & $57.7 \pm 13.3$ \\
& $\mathrm{~T}_{5}$ & $94.1 \pm 15.1^{\mathrm{a}, \mathrm{b}}$ & $59.5 \pm 14.9^{\mathrm{a}}$ & $24.9 \pm 7.6^{\mathrm{a}}$ \\
$\mathrm{S}$ & $\mathrm{T}_{0}$ & $39.7 \pm 11.6$ & $37.3 \pm 13.4$ & $49.2 \pm 23.9$ \\
& $\mathrm{~T}_{5}$ & $90.2 \pm 17.3^{\mathrm{a}, \mathrm{b}}$ & $53.9 \pm 8.7^{\mathrm{a}}$ & $25.2 \pm 4.9^{\mathrm{a}}$ \\
$\mathrm{ES}$ & $\mathrm{T}_{0}$ & $54.1 \pm 8.2$ & $42.9 \pm 13.9$ & $29.3 \pm 5.1$ \\
& $\mathrm{~T}_{5}$ & $56.2 \pm 19.9^{\mathrm{c}, \mathrm{d}}$ & $49.7 \pm 18.7$ & $30.5 \pm 13.5$ \\
\hline
\end{tabular}

Data are presented as the mean \pm standard deviation. ${ }^{\mathrm{a}} \mathrm{P}<0.05$ vs. $\mathrm{T}_{0}$; ${ }^{\mathrm{b}} \mathrm{P}<0.05$ vs. Group $\mathrm{C}$ at the same time; ${ }^{\mathrm{c}} \mathrm{P}<0.05$ vs. Group $\mathrm{H}$ at the same time; and ${ }^{d} \mathrm{P}<0.05$ vs. Group $\mathrm{S}$ at the same time. ALI, acute lung injury; IL, interleukin; $\mathrm{C}$, control group; $\mathrm{H}$, hypoxia group; $\mathrm{S}$, sevoflurane group; ES, combined sevoflurane-epidural anesthesia group; $\mathrm{T}_{0}$, spontaneous breathing; $\mathrm{T}_{5}, 180 \mathrm{~min}$ after ALI induction.

analyses. Total phospholipid (TPL), saturated phosphatidylcholine (SatPC) and total protein (TP) levels in the BALF were measured using Bartlett's (14), Mason's (15) and Lowry's (16) methods, respectively. The SatPC/TPL and SatPC/TP ratios represented the activity of pulmonary surfactant.

Statistical analysis. SPSS software, version 11.5 (SPSS, Inc., Chicago, IL, USA) was used for statistical analysis. Results are expressed as the mean \pm standard deviation. Data were compared using one-way analysis of variance and the $t$-test (Student-Newman-Keuls method). Non-normally distributed data were tested using Kruskal-Wallis analysis of variance. $\mathrm{P}<0.05$ was considered to indicate a statistically significant difference.

\section{Results}

Animal model establishment. Rabbit gender and weight were not significantly different between the groups $(\mathrm{P}>0.05)$. ALI models were induced at 15 min after hypoxia in Groups H, S and ES and was maintained at the standard of ALI during the entire process of hypoxia (Table I). The successful establishment of the TEA model with an epidural catheter placed into 
Table III. Relative mRNA expression levels of IL-6, IL-8 and IL-10 in the lung tissue (95\% confidence interval).

\begin{tabular}{llll}
\hline Group & \multicolumn{1}{c}{ IL-6 } & \multicolumn{1}{c}{ IL-8 } & \multicolumn{1}{c}{ IL-10 } \\
\hline $\mathrm{C}$ & $1.000(0.671-1.671)^{\mathrm{a}, \mathrm{b}}$ & $1.000(0.370-1.370)^{\mathrm{a}, \mathrm{b}}$ & $1.000(0.263-1.263)^{\mathrm{a}, \mathrm{b}}$ \\
$\mathrm{H}$ & $6.063(1.848-7.911)$ & $4.925(0.764-5.689)$ & $0.253(0.075-0.328)$ \\
$\mathrm{S}$ & $3.605(1.589-5.203)^{\mathrm{a}}$ & $5.401(2.328-7.730)$ & $0.287(0.092-0.379)$ \\
ES & $1.258(0.110-1.368)^{\mathrm{a}, \mathrm{b}}$ & $1.217(0.361-1.577)^{\mathrm{a}, \mathrm{b}}$ & $0.880(0.452-1.332)^{\mathrm{a}, \mathrm{b}}$ \\
\hline
\end{tabular}

IL, interleukin; C, control group; H, hypoxia group; S, sevoflurane group; ES, combined sevoflurane-epidural anesthesia group. ${ }^{\text {a }}<0.05$ vs. Group $\mathrm{H}$ at the same time. ${ }^{\mathrm{b}} \mathrm{P}<0.05$ vs. Group $\mathrm{S}$ at the same time.

Table IV. Pulmonary surfactant and D/W ratio changes after ALI induction.

\begin{tabular}{llll}
\hline Group & SatPC/TPL & SatPC/TP & \multicolumn{1}{c}{ D/W } \\
\hline C & $0.62 \pm 0.08$ & $0.67 \pm 0.29$ & $0.21 \pm 0.01$ \\
H & $0.44 \pm 0.14^{\mathrm{a}}$ & $0.28 \pm 0.11^{\mathrm{a}}$ & $0.17 \pm 0.02^{\mathrm{a}}$ \\
S & $0.31 \pm 0.13^{\mathrm{a}}$ & $0.14 \pm 0.04^{\mathrm{a}, \mathrm{b}}$ & $0.18 \pm 0.01$ \\
ES & $0.49 \pm 0.08^{\mathrm{a}, \mathrm{c}}$ & $0.29 \pm 0.16^{\mathrm{a}, \mathrm{c}}$ & $0.20 \pm 0.02$ \\
\hline
\end{tabular}

Data are presented as the mean \pm standard deviation. SatPC/TPL and SatPC/TP ratios represented the activity of pulmonary surfactant. ${ }^{\mathrm{a}} \mathrm{P}<0.05$ vs. Group $\mathrm{C} ;{ }^{\text {b }} \mathrm{P}<0.05$ vs. Group $\mathrm{H}$; and ${ }^{\mathrm{c}} \mathrm{P}<0.05$ vs. Group $\mathrm{S}$. $\mathrm{D} / \mathrm{W}$, dry weight/wet weight ratio of lung tissue; ALI, acute lung injury; SatPC, saturated phosphatidylcholine; TP, total protein; TPL, total phospholipid; $\mathrm{C}$, control group; $\mathrm{H}$, hypoxia group; $\mathrm{S}$, sevoflurane group; ES, combined sevoflurane-epidural anesthesia group.

the fifth or sixth thoracic spinous process was confirmed by epidurography (Fig. 1).

Serum cytokine concentrations and mRNA expression following hypoxia. The serum IL-6 and IL-8 levels were significantly higher in Groups $\mathrm{H}$ and $\mathrm{S}$ than in Group $\mathrm{C}$ while the IL-10 level in the two groups was significantly decreased at 3-h after the induction of ALI. Furthermore, there was significantly lower level of IL-6 in Group ES than in Groups $\mathrm{H}$ and S at this time (Table II).

Group H showed significantly higher IL-6 mRNA expression levels compared with the other three groups, and Group $\mathrm{S}$ showed significantly higher IL-6 mRNA expression compared with Groups $\mathrm{C}$ and $\mathrm{ES}(\mathrm{P}<0.05)$. Groups $\mathrm{H}$ and $\mathrm{S}$ showed significantly higher IL-8 mRNA expression compared with the other two groups $(\mathrm{P}<0.05)$; however, there was no significant difference between Groups $\mathrm{H}$ and $\mathrm{S}(\mathrm{P}>0.05)$. Group ES showed significantly higher IL-10 mRNA expression than Groups $\mathrm{H}$ and $\mathrm{S}(\mathrm{P}<0.05)$. Compared with Group C, Groups H and $\mathrm{S}$ showed significantly reduced IL-10 mRNA expression $(\mathrm{P}<0.05)$; however, no significant difference was detected between Groups $\mathrm{H}$ and $\mathrm{S}(\mathrm{P}>0.05$; Table III).

Pulmonary surfactants and the D/W ratio following hypoxia. The SatPC/TPL and SatPC/TP ratios were decreased in Groups H, S and ES compared with Group C. The SatPC/TP ratio in Group $\mathrm{S}$ was significantly reduced compared with those in Groups $\mathrm{H}$ and ES, while the SatPC/TPL ratio in Group $S$ was reduced compared with that in Group ES $(\mathrm{P}<0.05)$. Group $\mathrm{H}$ was the only group in which the $\mathrm{D} / \mathrm{W}$ ratio was significantly reduced compared with that of Group C ( $\mathrm{P}<0.05$; Table IV).

Light microscopy and transmission electron microscopy observations. The histopathological examination revealed severe hyperemia, exudate in a number of alveolar spaces, and obvious vascular expansion of the alveolar walls in Groups $\mathrm{H}$ and S. Bronchial epithelial cells appeared to be undergoing detachment, and pulmonary interstitial edema was evident, with a large quantity of neutrophil infiltration. The alveolar interval was increased and numerous alveoli were atelectatic. Reduced neutrophil infiltration and fewer collapsed alveoli were observed in Group ES compared with Groups $\mathrm{H}$ and $\mathrm{S}$ (Fig. 2). The alveolar type II cells in all groups contained numerous lamellar bodies (surfactant). In Groups $\mathrm{H}$ and $\mathrm{S}$ the alveolar type II cells were swollen, and the numbers of lamellar bodies and the amount of pulmonary surfactants within them were reduced compared with the other groups. The ultrastructure of the alveolar type II cells and of the capillary endothelium were comparable in Groups C and ES (Fig. 3).

\section{Discussion}

The present study investigated the potential effect of TEA on hypoxia-induced acute lung injury (ALI) using a rabbit model of ALI induced by exposure to acute hypoxia $\left(14 \% \mathrm{O}_{2}\right.$ in $\left.\mathrm{N}_{2}\right)$ at 15 min after intubation and mechanical ventilation.

ALI is a local pulmonary manifestation of the mononuclear macrophage systemic inflammatory response, which is caused by severe infection or trauma (17). It has previously been demonstrated that the mechanism of ALI involves the abnormal release of inflammatory mediators and a deficiency in the release of endogenous anti-inflammatory mediators (18). The imbalance between inflammatory and anti-inflammatory mediators aggravates the inflammatory response and promotes the development of ALI (19). In the present study, IL-6, IL-8 and IL-10 were selected for investigation, and the results showed that the levels of the inflammatory mediators IL-6 and IL-8 and the anti-inflammatory mediator IL-10 changed in all groups following ALI induction.

IL-6 is produced rapidly following tissue damage and is the primary proinflammatory cytokine responsible for inducing the systemic changes known as the acute-phase response. The IL-6 
A

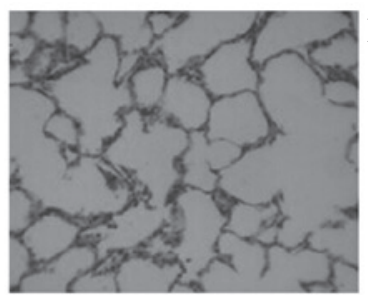

$\mathbf{E}$

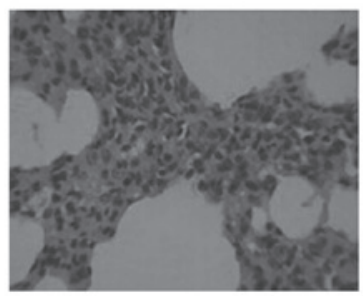

B

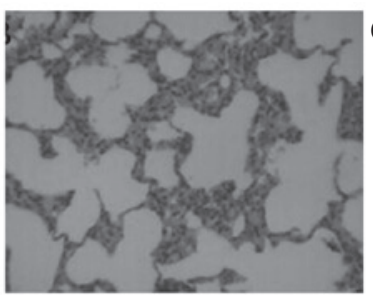

F

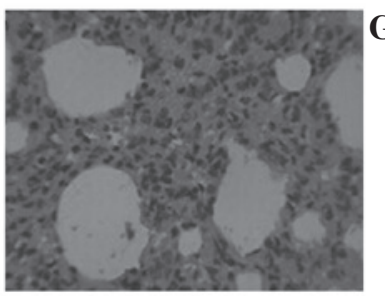

C
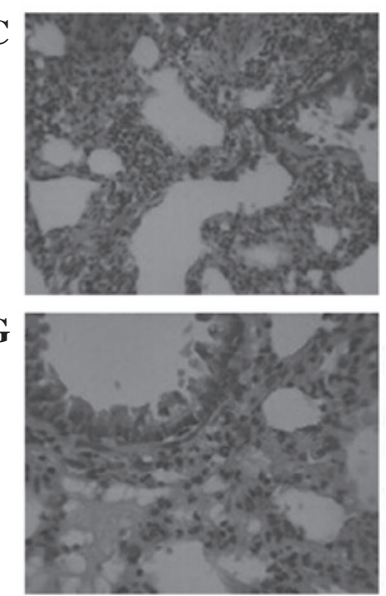

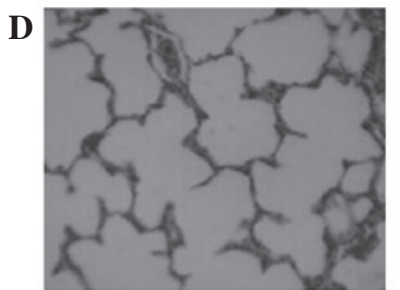

Figure 2. Representative light microscopy images, stained with hematoxylin and eosin. (A) Group C, the control group; (B) Group H, hypoxia group; (C) Group S, sevoflurane group; and (D) Group ES, combined sevoflurane-epidural anesthesia group (A-D magnification, x200). (E) Group H, (F) Group S and (G) Group ES at x400 magnification.
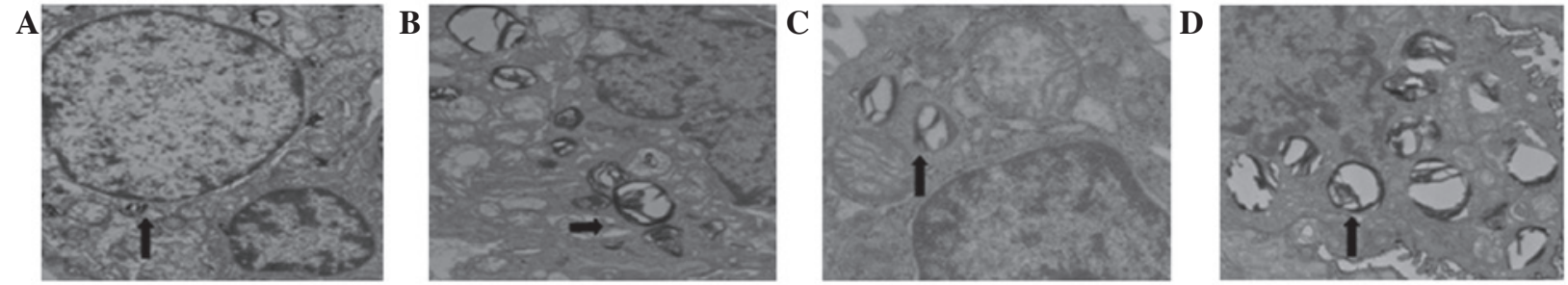

Figure 3. Transmission electron microscopy results (magnification, x18,000). Images show (A) swollen alveolar type II cells in Group S after $3 \mathrm{~h}$ hypoxia, (B) lamellar bodies in Group C, (C) decreased lamellar bodies in Group S and (D) lamellar bodies in Group ES. Group C, control group; Group H, hypoxia group; Group S, sevoflurane group; Group ES, combined evoflurane-epidural anesthesia group.

level provides an accurate indication of the severity of systemic inflammatory response syndrome (20). It has previously been demonstrated that systemic and local lung tissue levels of IL-6 are increased during invasive mechanical ventilation (21). The present study showed that the IL-6 levels in Groups H and S were significantly higher compared with those in Groups ES and C, and that IL-6 levels increased compared with baseline following ALI induction. These results indicate that IL- 6 functions as a mediator of the inflammatory cascade reaction in ALI, and that TEA is able to effectively mitigate the increase in the IL-6 level, which may be associated with sympathetic nerve blockade and the reduction of nociceptive transmission. The present results are consistent with the findings of a previous study (22).

IL-8 is an inflammatory chemokine that serves a crucial function in the regulation of inflammation and immunity. A clinical trial including 27 patients that underwent an esophagectomy showed that the level of IL-8 in the peripheral blood and chest drainage liquid in 11 patients with ALI was significantly increased compared with that in the 16 patients without ALI (23). In the present study, acute hypoxia led to an clear increase in the IL- 8 level in Group S. According to previous studies (24), IL-8 participates in the development of ALI by influencing the biological activity of granulocytes via polymorphonuclear leukocytes (25).

IL-10 is produced by various inflammatory cells, especially macrophages, and exhibits multifaceted anti-inflammatory properties, including the inhibition of matrix degrading metalloproteinase production, leading to suppressed cytokine production, reduction of tissue factor expression and inhibition of the apoptosis of macrophages and monocytes after infection. These inflammatory mechanisms have been demonstrated to contribute to atherosclerotic lesion development and progression, indicating a potential regulatory role of IL-10 (26). Sevoflurane is an inhalational anesthetic that is widely used in clinical practice. Goto et al (27) showed that sevoflurane had no influence on neutrophil apoptosis, nor on IL-6 or IL-8 concentrations. Furthermore, El Azab et al (28) showed that sevoflurane did not significantly affect inflammatory cells. In the present study, compared with baseline, the serum IL-6 and IL-8 levels in Groups $\mathrm{H}$ and S significantly increased, while the IL-10 level significantly decreased following ALI. The imbalance between proinflammatory and anti-inflammatory factors may underlie the ALI-associated tissue damage. However, the lack of a significant difference between Groups $\mathrm{H}$ and $\mathrm{S}$, suggests that sevoflurane has no clear influence on inflammation during acute hypoxia. This may be due to the relative brevity of the hypoxic period. Furthermore, the serum IL-6 level in Group ES was significantly reduced compared those in Groups $\mathrm{H}$ and $\mathrm{S}$, suggesting that epidural anesthesia inhibited the systemic inflammatory response to a certain extent.

ALI affects the systemic inflammatory response in addition to the local inflammation of lung tissue. The present study observed that the mRNA expression levels of IL-6, IL-8 and IL-10 changed in the four groups following acute hypoxia, 
and the changes were consistent with a previous study by Kalenka et al (20), suggesting that epidural anesthesia exerted a protective effect. Although epidural anesthesia cannot completely inhibit systemic and local inflammation, it may be able to decrease the severity of reactions.

Pulmonary surfactants have unique physiological effects in maintaining the normal breathing function. A previous study indicated that inhalational anesthetics have a direct impact on pulmonary surfactants; the inhalation of fat-soluble anesthetics increased alveolar surface tension, which was unchanged following the inhalation of non-fat-soluble anesthetics (29). The higher surface tension increased the release and synthesis of the primary active components, such as phosphatidylcholine (30). It has been demonstrated that phosphatidylglycerol is able to maintain the structural integrity of lipid-protein complexes, promote membrane activity and stability and increase membrane fluidity (31). Furthermore, it has been reported that purine metabolites in alveolar lavage fluid may be used as an index to judge the severity of lung injury (32). In order to correct the deviation caused by the dilution difference between alveolar lavage and phosphatidylcholine, SatPC/TPL and SatPC/TP ratios were used in the present study as indices of phosphatidylcholine activity. The results showed that the SatPC/TPL and SatPC/TP ratios in Groups $\mathrm{H}, \mathrm{S}$ and ES were significantly decreased compared with those in Group C, which additionally demonstrated the successful induction of the ALI model. The SatPC/TP ratio in Group $\mathrm{S}$ was decreased compared with those in Groups H and ES, while the SatPC/TPL ratio was lower compared with that in Group ES, suggesting that sevoflurane reduced the activity of the pulmonary surfactants. The electron microscopy results were consistent with this phenomenon.

Animal experiments have shown that following the administration of inhalation anesthetics, the main change in alveolar type II cell ultrastructure is that involving the lamellar bodies (33). Lamellar bodies constitute the storage pool of pulmonary surfactants. Changes in the lamellar body number and volume indicate the transportation of pulmonary surfactants. Following the administration of inhalational non-fat-soluble anesthetics, lamellar body exocytosis increases. However, the volume density and number density remain unchanged, suggesting that the synthesis and secretory function of surface-active substances by alveolar type II cells are not obviously influenced (34). Following the inhalation of fat-soluble anesthetics, exocytosis of lamellar bodies is rare; however, the volume density and number density are significantly increased, suggesting that the secretory function of alveolar type II cells is disturbed while synthesis is not influenced (35). Therefore, the surface-active substance becomes isolated within the cell. In the present study, the morphological observations were consistent with the quantitative test results.

In the present study, the alveolar type II cells appeared round, oval or polygonal under transmission electron microscopy. The membranes contained microvilli and features of the nucleus, mitochondria and lamellar bodies were observable inside the cells. Certain cells were rich in endoplasmic reticulum. Surface-active substances were adherent to the alveolar cavity wall, rendering a slightly higher electron density at the interface. The alveolar cavity contained macrophagocytes, and occasionally exhibited a paracrystalline appearance, which represented the absence of surface-active substance movement. Furthermore, the alveolar type II epithelial cells were swollen in Groups $\mathrm{H}$ and S. Microvilli were shortened and reduced in number. No obvious changes were observed in Group ES. There were fewer lamellar bodies in Group S, and they demonstrated a large quantity of vacuolation; however, the lamellar bodies were not clearly reduced in Group ES, suggesting that the primary effect of sevoflurane on alveolar type II cells was on the lamellar bodies.

The present study had a limited sample size, and so further extended analysis with a larger sample size or an independent replication study is required to confirm the positive association of TEA with ALI. ALI is a complex pathophysiological process; therefore, the mechanism underlying the impact of epidural anesthesia on ALI remains unclear and requires further investigation.

In conclusion, the present study investigated the effects of TEA on function and morphology in rabbits exposed to hypoxia-induced lung injury. The results indicated that TEA serves a crucial function in reducing systemic and local inflammation, decreasing the interference of inhaled anesthetics with pulmonary surfactants and improving the alveolar ultrastructure.

\section{References}

1. Reiss LK, Uhlig U and Uhlig S: Models and mechanisms of acute lung injury caused by direct insults. Eur J Cell Biol 91: 590-601, 2012 .

2. Rubenfeld GD, Caldwell E, Peabody E, Weaver J, Martin DP Neff M, Stern EJ and Hudson LD: Incidence and outcomes of acute lung injury. N Engl J Med 353: 1685-1693, 2005.

3. Jain $\mathrm{S}$ and Bellingan G: Basic science of acute lung injury. Surgery 25: 112-116, 2007.

4. Rassler B, Marx G, Reissig C, Rohling MA, Tannapfel A, Wenger RH and Zimmer HG: Time course of hypoxia-induced lung injury in rats. Respir Physiol Neurobiol 159: 45-54, 2007.

5. Bhargava $\mathrm{M}$ and Wendt $\mathrm{CH}$ : Biomarkers in acute lung injury. Transl Res 159: 205-217, 2012

6. Cross LJ and Matthay MA: Biomarkers in acute lung injury: Insights into the pathogenesis of acute lung injury. Crit Care Clin 27: 355-377, 2011.

7. Dombrowsky H, Barrenschee M, Kunze $\mathbf{M}$ and Uhlig S: Conserved responses to trichostatin $\mathrm{A}$ in rodent lungs exposed to endotoxin or stretch. Pulm Pharmacol Ther 22: 593-602, 2009.

8. Calfee CS, Eisner MD, Ware LB, Thompson BT, Parsons PE, Wheeler AP, Korpak A and Matthay MA; Acute Respiratory Distress Syndrome Network, National Heart, Lung, and Blood Institute: Trauma-associated lung injury differs clinically and biologically from acute lung injury due to other clinical disorders. Crit Care Med 35: 2243-2250, 2007.

9. Reyal Y and Bellingan G: The basic science of acute lung injury. Surgery 22: iii-vii, 2004.

10. Schwarzkopf K, Schreiber T, Bauer R, Schubert H, Preussler NP, Gaser E, Klein U and Karzai W: The effects of increasing concentrations of isoflurane and desflurane on pulmonary perfusion and systemic oxygenation during one-lung ventilation in pigs. Anesth Analg 93: 1434-1438, 2001.

11. Onan IS, Onan B, Korkmaz AA, Oklu L, Kilickan L, Gonca S, Dalcik $H$ and Sanisoglu I: Effects of thoracic epidural anesthesia on flow and endothelium of internal thoracic artery in coronary artery bypass graft surgery. J Cardiothorac Vasc Anesth 25: 1063-1070, 2011.

12. Abdelrahman RS: Effects of thoracic epidural anesthesia on pulmonary venous admixture and oxygenation with isoflurane or propofol anesthesia during one lung ventilation. Egyptian J Chest Dis Tuberc 61: 477-483, 2012.

13. Kaneda H, Waddell TK, de Perrot M, Bai XH, Gutierrez C, Arenovich T, Chaparro C, Liu $M$ and Keshavjee S: Pre-implantation multiple cytokine mRNA expression analysis of donor lung grafts predicts survival after lung transplantation in humans. Am J Transplant 6: 544-551, 2006.

14. Bartlett GR: Phosphorous assay in column chromatography. J Biol Chem 234: 466-468, 1959. 
15. Mason RJ, Nellenbogen $\mathrm{J}$ and Clements JA: Isolation of disaturated phosphatidylcholine with osmium tetroxide. J Lipid Res 17: 281-284, 1976.

16. Magnusson OT, Toyama H, Saeki M, Schwarzenbacher R and Klinman JP: The structure of a biosynthetic intermediate of pyrroloquinoline quinine (PQQ) and elucidation of the final step of PQQ biosynthesis. J Am Chem Soc 126: 5342-5343, 2004.

17. Bein T, Zimmermann M, Schiewe-Langgartner F, Strobel R, Hackner K, Schlitt HJ, Nerlich MN, Zeman F, Graf BM and Gruber M: Continuous lateral rotational therapy and systemic inflammatory response in posttraumatic acute lung injury: Results from a prospective randomised study. Injury 43: 1892-1897, 2012

18. Goodman RB, Pugin J, Lee JS and Matthay MA: Cytokine-mediated inflammation in acute lung injury. Cytokine Growth Factor Rev 14: 523-535, 2003.

19. Fu PK, Yang CY, Tsai HT and Hsieh CL: Moutan cortex radicis improves lipopolysaccharide-induced acute lung injury in rats through anti-inflammation. Phytomedicine 19: 1206-1215, 2012.

20. Kalenka A, Feldmann RE Jr, Otero K, Maurer MH, Waschke KF and Fiedler F: Changes in the serum proteome of patients with sepsis and septic shock. Anesth Analg 103: 1522-1526, 2006.

21. Van Wessem KJ, Hennus MP, Heeres M, Koenderman L and Leenen LP: Mechanical ventilation is the determining factor in inducing an inflammatory response in a hemorrhagic shock model. J Surg Res 180: 125-132, 2013.

22. Yokoyama M, Itano $\mathrm{Y}$, Katayama H, Morimatsu H, Takeda Y, Takahashi T, Nagano $\mathrm{O}$ and Morita K: The effects of continuous epidural anesthesia and analgesia on stress response and immune function in patients undergoing radical esophagectomy. Anesth Analg 101: 1521-1527, 2005.

23. Morita M, Yoshida R, Ikeda K, Egashira A Oki E, Sadanaga N, Kakeji Y, Ichiki Y, Sugio K, Yasumoto K and Maehara Y: Acute lung injury following an esophagectomy for esophageal cancer, with special reference to the clinical factors and cytokine levels of peripheral blood and pleural drainage fluid. Dis Esophagus 21: 30-36, 2008.

24. Callister ME, Burke-Gaffney A, Quinlan GJ, Nicholson AG, Florio R, Nakamura H, Yodoi J and Evans TW: Extracellular thioredoxin levels are increased in patients with acute lung injury. Thorax 61: 521-527, 2006.
25. Misumi T, Tanaka T, Mikawa K, Nishina K, Morikawa $\mathrm{O}$ and Obara H: Effects of sivelestat, a new elastase inhibitor, on IL-8 and MCP-1 production from stimulated human alveolar epithelial type II cells. J Anesth 20: 159-165, 2006.

26. Li JJ, Guo YL and Yang YJ: Enhancing anti-inflammatory cytokine IL-10 may be beneficial for acute coronary syndrome. Med Hypotheses 65: 103-106, 2005.

27. Goto Y, Ho SL, McAdoo J, Fanning NF, Wang J, Redmond HP and Shorten GD: General versus regional anaesthesia for cataract surgery: Effects on neutrophil apoptosis and the postoperative pro-inflammatory state. Eur J Anaesthesiol 17: 474-480, 2000.

28. El Azab SR, Rosseel PM, De Lange JJ, van Wijk EM, van Strik R and Scheffer GJ: Effect of VIMA with sevoflurane versus TIVA with propofol or midazolam-sufentanil on the cytokine response during CABG surgery. Eur J Anaesthesiol 19: 276-282, 2002.

29. Southorn P, Rehder K and Hyatt RE: Halothane anesthesia and respiratory mechanics in dogs lying supine. J Appl Physiol Respir Environ Exerc Physiol 49: 300-305, 1980.

30. Zuo YY, Veldhuizen RA, Neumann AW, Petersen NO and Possmayer F: Current perspectives in pulmonary surfactant-inhibition, enhancement and evaluation. Biochim Biophys Acta 1778: 1947-1977, 2008.

31. Agassandian M and Mallampalli RK: Surfactant phospholipid metabolism. Biochim Biophys Acta 1831: 612-625, 2013.

32. Verbrugge SJ, de Jong JW, Keijzer E, Vazquez de Anda G and Lachmann B: Purine in bronchoalveolar lavage fluid as a marker of ventilation-induced lung injury. Crit Care Med 27: 779-783, 1999.

33. Southorn P, Rehder K and Hyatt RE: Halothane anesthesia and respiratory mechanics in dogs lying supine. J Appl Physiol Respir Environ Exerc Physiol 49: 300-305, 1980.

34. Evans JA, Hamilton RW Jr, Kuenzig MC and Peltier LF: Effects of anesthetic agents on surface properties of dipalmitoyllecithin: Lung surfactant model. Anesth Analg 45: 285-289, 2002

35. Song J, Lin G and Guo S: Effects of inhalational anesthetic agents on ultrastructure of the type II alveolar cell. Acta Academiae Medicinae Nanjing 22: 497-499, 2004 (In Chinese) 